\title{
Impact of slow-light enhancement on optical propagation in active semiconductor photonic crystal waveguides
}

Chen, Yaohui; de Lasson, Jakob Rosenkrantz; Gregersen, Niels; Mørk, Jesper

Published in:

Physical Review A

Link to article, DOI:

10.1103/PhysRevA.92.053839

Publication date:

2015

Document Version

Publisher's PDF, also known as Version of record

Link back to DTU Orbit

Citation (APA):

Chen, Y., de Lasson, J. R., Gregersen, N., \& Mørk, J. (2015). Impact of slow-light enhancement on optical propagation in active semiconductor photonic crystal waveguides. Physical Review A, 92, 8. [053839]. https://doi.org/10.1103/PhysRevA.92.053839

\section{General rights}

Copyright and moral rights for the publications made accessible in the public portal are retained by the authors and/or other copyright owners and it is a condition of accessing publications that users recognise and abide by the legal requirements associated with these rights.

- Users may download and print one copy of any publication from the public portal for the purpose of private study or research.

- You may not further distribute the material or use it for any profit-making activity or commercial gain

- You may freely distribute the URL identifying the publication in the public portal 


\title{
Impact of slow-light enhancement on optical propagation in active semiconductor photonic-crystal waveguides
}

\author{
Yaohui Chen, Jakob Rosenkrantz de Lasson, Niels Gregersen, and Jesper Mørk* \\ DTU Fotonik, Department of Photonics Engineering, Technical University of Denmark, Ørsteds Plads, Building 343, \\ DK-2800 Kongens Lyngby, Denmark \\ (Received 21 August 2015; published 17 November 2015)
}

\begin{abstract}
We derive and validate a set of coupled Bloch wave equations for analyzing the reflection and transmission properties of active semiconductor photonic-crystal waveguides. In such devices, slow-light propagation can be used to enhance the material gain per unit length, enabling, for example, the realization of short optical amplifiers compatible with photonic integration. The coupled-wave analysis is compared to numerical approaches based on the Fourier modal method and a frequency domain finite-element technique. The presence of material gain leads to the buildup of a backscattered field, which is interpreted as distributed feedback effects or reflection at passive-active interfaces, depending on the approach taken. For very large material gain values, the band structure of the waveguide is perturbed, and deviations from the simple coupled Bloch wave model are found.
\end{abstract}

DOI: 10.1103/PhysRevA.92.053839

PACS number(s): 42.55.Px, 42.55.Tv, 42.70.Qs

\section{INTRODUCTION}

Photonic-crystal $(\mathrm{PhC})$ structures have been proposed as a waveguide infrastructure for high-density photonic integrated circuits [1-3]. Optical amplification is one of the fundamental functionalities required for compensating attenuation and coupling losses and thus scaling up the number of integrated devices [4]. A major advantage of using photonic-crystal waveguides, as opposed to standard ridge-type waveguides, when realizing active structures with embedded semiconductor gain layers is the use of slow-light effects to enhance lightmatter interactions. Thus, the spatial gain coefficient may be increased by exploiting slow-light propagation, enabling the realization of short devices suitable for photonic integration [5-7]. Similarly, nonlinear effects induced by carrier depletion may also be enhanced, leading to devices with ultralow saturation power, which may be of interest for optical signal processing applications [8].

In this work, we theoretically investigate the frequencydomain optical propagation properties of an active $\mathrm{PhC}$ waveguide of finite length embedded in an ideal passive periodic PhC waveguide platform as shown in Fig. 1. Such structures with site-controlled active gain sections may be fabricated using different techniques [9-12].

As shown in Fig. 1, we approximate the device as a photonic-crystal heterostucture [13] with a slowly varying envelope of the imaginary part of the refractive index (bottom part) and a fast periodic variation that naturally arises from the penetration of the air holes through the gain layer (top part). Such an envelope approximation in photonic-crystal devices is analogous to the treatment of semiconductor optoelectronic devices. Based on a perturbative approach [14], effective one-dimensional (1D) coupled-wave analysis has been widely used to investigate the impact of slow-light effects on optical properties of passive $\mathrm{PhC}$ waveguides, e.g., for efficient taper design [15], Kerr nonlinearities [16,17], and disorder-induced scattering [18,19].

\footnotetext{
*jesm@fotonik.dtu.dk
}

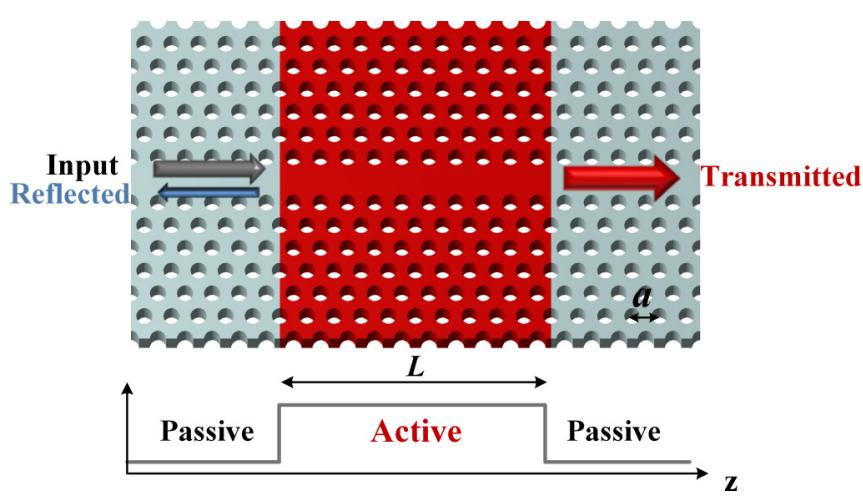

FIG. 1. (Color online) Illustration of defect photonic-crystal waveguide. The red part of the structure is active, i.e., this part of the membrane structure contains embedded layers of quantum wells or quantum dots that may provide gain upon optical or electrical pumping. The lattice constant is $a$ and the length of the active region is $L$.

For perfectly periodic $\mathrm{PhC}$ waveguides, neglecting Kerr nonlinearity and disorder, a rigorous set of equations for the amplitudes of forward- and backward-propagating unperturbed Bloch waves may be derived [20]. In such a formulation, the presence of active material in a finite section of the $\mathrm{PhC}$ waveguide leads to multiple scattering, which represents material-gain-induced coupling between the forward and backward Bloch waves of passive structures. Such distributed feedback (DFB) effects have so far not been considered in slow-light enhanced semiconductor optical amplifiers [7,21-23]. We show here that it is important to consider the impact of such feedback effects when calculating the gain of an active $\mathrm{PhC}$ waveguide.

Alternatively, we may treat the structure as multiple waveguide sections, namely an active $\mathrm{PhC}$ waveguide section interfaced with two semi-infinite passive $\mathrm{PhC}$ waveguides on both sides, with distinct sets of Bloch modes in the passive and active $\mathrm{PhC}$ waveguide sections. In this picture, the active-passive interfaces induce reflections, and Fabry-Pérot effects are important in determining the strength of the 
signal transmitted from the input to the output waveguide as well as the back-reflected signal. We solve the optical multiple-scattering problem in aggregates of generalized thin films using the Fourier modal method (FMM) [or rigorous coupled wave analysis (RCWA)] [24-26], which has been widely implemented for various periodic photonic structures. Using these methods, Bloch waves are computed and sorted in both passive and active $\mathrm{PhC}$ sections [27].

In the following, we numerically validate that both approaches are equivalent in analyzing the proposed active PhC devices. A similar discussion for active Bragg grating structures can be found in [28]. We also analyze the situation with very large material gain values, in which case the band structure of the photonic-crystal structure is modified.

Here we limit our attention to the simplest type of photoniccrystal waveguide, with one row of missing holes (W1 waveguide). In that case, slow-light propagation is realized close to the Brillouin zone edge and the bandwidth over which significant slowdown effects are realized is limited. It was shown, though, that by dispersion engineering the waveguide, a slow-light region of significant bandwidth can be obtained for a frequency range displaced from the band edge [29]. A systematic approach for optimizing the group index bandwidth product was recently demonstrated [30]. For such dispersion-engineered structures, the large group index region may be centered around an inflection point of the dispersion curves and it was shown that strong loss (or gain) can induce drastic changes of the dispersion curves [31].

The paper is organized as follows: In Sec. II we derive the coupled Bloch wave equations. Section III presents the numerical results. We first consider a long waveguide with relatively weak gain, and compare the coupled-wave analysis to numerical results obtained using the FMM. Second, we consider a shorter waveguide, enabling the consideration of larger absolute gain coefficients as well as the use of a finiteelement numerical technique. Finally, the main conclusions are summarized in Sec. IV.

\section{EFFECTIVE ONE-DIMENSIONAL COUPLED-WAVE ANALYSIS: PERTURBATION AND DISTRIBUTED FEEDBACK}

We consider a line-defect photonic-crystal waveguide, where part of the waveguide is active, as illustrated in Fig. 1. The active material may be implemented as layers of quantum wells or quantum dots embedded in the middle of the photoniccrystal membrane. In order to limit the energy consumption, it is preferable only to have active material in the core of the waveguide, as demonstrated in [9]. Here, however, we consider the simpler structure, where the membrane is uniformly active in the transverse directions $(x y)$. This is appropriate for the case of optically pumped structures, as considered, for example, in [7]. We represent the material gain as a weak perturbation to the passive periodic $\mathrm{PhC}$ waveguide in a finite-length section and use the Bloch modes of the passive structure to expand the field of the structure including gain. In this formulation, multiple scattering in the active PhC structure is represented as material-gain-induced coupling between the forward- and backward-propagating Bloch waves of the passive structure,

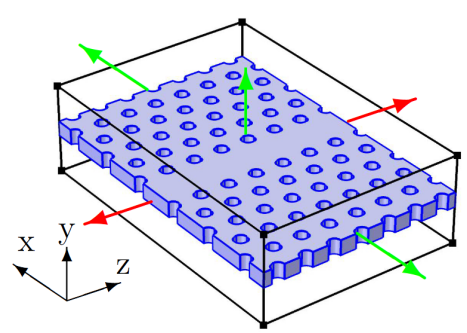

(a)

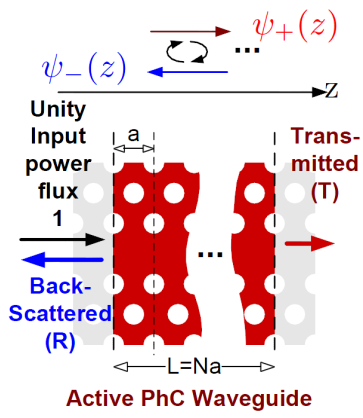

(b)
FIG. 2. (Color online) Illustration of coupled-wave analysis of $\mathrm{PhC}$ waveguide. (a) Schematic of surface used in the derivation of coupled mode equations, with arrows indicating outward unit normal vectors to the boundary surface. (b) Schematic diagram of coupling between the amplitudes of forward- and backward-propagating Bloch waves induced by the active material, which is represented by an imaginary perturbation of the refractive index.

as illustrated in the top part of Fig. 2(b). This is similar to the way disorder is analyzed in [18].

In the continuous wave (cw) limit, the Maxwell equations can be rewritten based on the conjugated form of the Lorentz reciprocity theorem $[17,32]$ :

$$
\nabla \cdot\left(\mathbf{E} \times \mathbf{H}_{0, \pm}^{*}+\mathbf{E}_{0, \pm}^{*} \times \mathbf{H}\right)=i \omega \mathbf{P}_{\text {pert }} \cdot \mathbf{E}_{0, \pm}^{*} .
$$

Here, $\mathbf{E}_{0, \pm}$ and $\mathbf{H}_{0, \pm}$ are the guided Bloch wave solutions of Maxwell equations for the electrical and magnetic fields obtained in the absence of electronic polarization perturbations $\mathbf{P}_{\text {pert }}$, with \pm denoting forward- or backward-propagating fields. The fields $\mathbf{E}$ and $\mathbf{H}$ are the self-consistent solutions in the presence of electronic polarization perturbations, which arise due to the presence of active material, e.g., leading to stimulated emission and thus gain of the propagating fields. We may represent the unperturbed Bloch waves as follows:

$$
\begin{aligned}
\mathbf{E}_{0, \pm} & =\frac{1}{2} \mathbf{e}_{ \pm}(x, y, z) \exp \left( \pm i k_{z} z\right) \exp (-i \omega t), \\
\mathbf{H}_{0, \pm} & =\frac{1}{2} \mathbf{h}_{ \pm}(x, y, z) \exp \left( \pm i k_{z} z\right) \exp (-i \omega t),
\end{aligned}
$$

with $\mathbf{e}_{ \pm}(x, y, z)$ and $\mathbf{h}_{ \pm}(x, y, z)$ being the normalized complex amplitude Bloch functions, $\omega$ the frequency, and $k_{z}$ the Bloch wave number along the length of the waveguide.

The unit rms power flux $P_{z}$ and unit rms electric and magnetic stored energy $W$ in a supercell are given as

$$
\begin{gathered}
P_{z}=\frac{1}{2} \int_{S}\left[\operatorname{Re}\left\{\mathbf{e} \times \mathbf{h}^{*}\right\} \cdot \hat{\mathbf{z}}\right] d S \\
W=\frac{1}{4} \int_{V}\left[\varepsilon_{0} n_{b}^{2}(\mathbf{r})|\mathbf{e}|^{2}+\mu_{0}|\mathbf{h}|^{2}\right] d V=\frac{a n_{g z}}{c} P_{z} .
\end{gathered}
$$

Here, $n_{g z} \equiv c / v_{g z}$ is the group index, where $c$ and $v_{g z}=$ $\partial \omega / \partial k_{z}$ are the speed of light and the group velocity, $\varepsilon_{0}$ and $\mu_{0}$ are the vacuum permittivity and permeability, $n_{b}(\mathbf{r})$ is the background refractive index, $a$ is the lattice constant of the photonic crystal, $S$ indicates the transverse plane at position $z$, and $V$ is the volume of a $\mathrm{PhC}$ supercell. 
In the limit of a weak electronic perturbation, we express the self-consistent solutions as superpositions of the guided Bloch waves for the passive structure, with slowly varying amplitudes $\psi_{ \pm}(z)$ :

$$
\begin{aligned}
& \mathbf{E} \simeq \psi_{+}(z) \mathbf{E}_{0,+}+\psi_{-}(z) \mathbf{E}_{0,-}, \\
& \mathbf{H} \simeq \psi_{+}(z) \mathbf{H}_{0,+}+\psi_{-}(z) \mathbf{H}_{0,-} .
\end{aligned}
$$

By using Eqs. (2), (3), (6), and (7), Eq. (1) can be formulated as a set of continuity equations for the forward- and backwardpropagating amplitudes:

$$
\pm \frac{1}{2} \nabla \cdot\left[\psi_{ \pm}(z) \operatorname{Re}\left\{\mathbf{e} \times \mathbf{h}^{*}\right\}\right]=i \omega \mathbf{P}_{\text {pert }} \cdot \mathbf{E}_{0, \pm}^{*} .
$$

Equation (8) can be derived using the divergence theorem for a closed surface $S$ enclosing the simulation domain:

$\pm \frac{1}{2} \oiiint_{S}\left[\psi_{ \pm}(z) \operatorname{Re}\left\{\mathbf{e} \times \mathbf{h}^{*}\right\} \cdot \hat{\mathbf{n}}\right] d S=i \omega \int_{V}\left(\mathbf{P}_{\mathbf{p e r t}} \cdot \mathbf{E}_{0, \pm}^{*}\right) d V$.

Here, $\hat{\mathbf{n}}$ is the outward unit normal vector of the closed surface depicted in Fig. 2(a). This closed surface can be chosen conveniently to comply with the experimental geometry considered (near-field measurement [33] or transmission measurement [34]), as well as the simulation domain and boundary conditions (BCs) (e.g., periodic BCs [35] or perfectly matched layer (PML) BCs [25]). The left-hand side (LHS) of Eq. (8) describes the possible channels for the power flux in or out of the surface and the right-hand side (RHS) describes the source term. Equation (9) is in the form of a set of implicit integral equations. Assuming that the considered modes are transversely confined, the surface integral [LHS of Eq. (9)] reduces to two integrals between the planes $z=0$ and $z=L$, described as

$$
\begin{aligned}
& \pm \frac{1}{2} \oiint_{S}\left[\psi_{ \pm}(z) \operatorname{Re}\left\{\mathbf{e} \times \mathbf{h}^{*}\right\} \cdot \hat{\mathbf{n}}\right] d S \\
& \quad \simeq \pm \int_{0}^{L} \partial_{z} \psi_{ \pm}(z) d z \times \frac{1}{2} \int_{S}\left[\operatorname{Re}\left\{\mathbf{e} \times \mathbf{h}^{*}\right\} \cdot \hat{\mathbf{z}}\right] d S .
\end{aligned}
$$

For simplicity, we describe the carrier-induced complex susceptibility perturbation as a product of a complex constant $\chi_{\text {pert }}$ and an active material distribution function $F(\mathbf{r})$ :

$$
\mathbf{P}_{\text {pert }}=\frac{1}{2} \varepsilon_{0} \chi_{\text {pert }} F(\mathbf{r}) \mathbf{E},
$$

where $F(\mathbf{r})=1(=0)$ in the active (passive) region.

We now obtain the coupled-mode equations from Eq. (9) with substitution from Eqs. (10) and (11):

$$
\begin{aligned}
& \partial_{z} \psi_{+}(z)=\frac{i \omega}{c} n_{g z} \chi_{\text {pert }}\left[\delta(z) \psi_{+}+\kappa^{*}(z) e^{-i 2 k_{z} z} \psi_{-}\right], \\
& \partial_{z} \psi_{-}(z)=-\frac{i \omega}{c} n_{g z} \chi_{\text {pert }}\left[\delta(z) \psi_{-}+\kappa(z) e^{i 2 k_{z} z} \psi_{+}\right],
\end{aligned}
$$

with

$$
\begin{gathered}
\delta(z) \equiv \frac{a \int_{S}\left[\varepsilon_{0} F(\mathbf{r})|\mathbf{e}|^{2}\right] d S}{\int_{V}\left[\epsilon_{0} n_{b}^{2}(\mathbf{r})|\mathbf{e}|^{2}+\mu_{0}|\mathbf{h}|^{2}\right] d V}, \\
\kappa(z) \equiv \frac{a \int_{S}\left[\varepsilon_{0} F(\mathbf{r}) \mathbf{e} \cdot \mathbf{e}\right] d S}{\int_{V}\left[\epsilon_{0} n_{b}^{2}(\mathbf{r})|\mathbf{e}|^{2}+\mu_{0}|\mathbf{h}|^{2}\right] d V} .
\end{gathered}
$$

Here, $\delta(z)$ and $\kappa(z)$ denote the complex-valued propagation and backscattering coefficient induced by the perturbation due to the active material. The above $1 \mathrm{D}$ coupled-wave equations are mathematically equivalent to a $2 \times 2$ scattering matrix problem in a stack of thin films (at each $z$ coordinate) and readily solved numerically.

We note the similarity of the above coupled-mode equations with the coupled-mode equations originally derived by Kogelnik and Shank for distributed feedback lasers [36]. However, while those equations describe the slow variation of the complex amplitudes of plane waves (see also [37] for application to $\mathrm{PhC}$ waveguides), Eqs. (12) and (13) describe the slowly varying amplitudes of the Bloch waves of the corresponding passive $\mathrm{PhC}$. This has the consequence that in the absence of a polarization perturbation, i.e., $\chi_{\text {pert }}=0$, the forward and backward Bloch waves do not couple but remain unidirectionally propagating waves, as required. The presence of a perturbation, however, leads to coupling of the Bloch waves, since the material perturbation gives rise to distributed feedback. This is analogous to the scattering between Bloch waves induced by structural disorder [18], although in that case the backscattering sites are randomly distributed.

In general, the harmonic term $\exp \left( \pm i 2 k_{z} z\right)$ leads to relatively low backscattering efficiency due to phase mismatch between forward- and backward-propagating Bloch waves. Propagation losses decrease the effective propagation length and further damp the backscattering. In passive $\mathrm{PhC}$ waveguides, one can integrate similar coupled-mode equations approximately by neglecting the distributed feedback terms as long as the backscattered power is weak [15]. On the other hand, in the case of positive gain, the effective length, over which wave interaction takes place, increases, and a significant backscattered wave may be built up, as we show here.

Equations (12) and (13) must be supplemented by boundary conditions at the passive-active interfaces. The boundary conditions [38] are

$$
\begin{gathered}
\psi_{+}(0)=r_{1} \psi_{-}(0)+\psi_{0}, \\
\psi_{-}(L) \exp \left(-i k_{z} L\right)=r_{2} \psi_{+}(L) \exp \left(i k_{z} L\right),
\end{gathered}
$$

where $r_{1}$ and $r_{2}$ are the amplitude reflectivities of the left and right passive-active interfaces and $\psi_{0}=1$ indicates unity incident field from the left interface.

Finally, we note that the coupled-wave approach presented here permits the inclusion of disorder-induced losses [18,19] and carrier dynamics [8], which is beyond the scope of this paper.

\section{SIMULATION RESULTS}

For the numerical investigations, we consider a twodimensional (2D) triangular lattice of air holes (hole radius $r=0.25 a$ ) embedded in a semiconductor membrane with a dielectric constant of $n_{\mathrm{s}}^{2}=12.1$. The waveguide is a W1 line defect with a single row of air holes omitted. The presence of material gain or absorption is modeled via an imaginary refractive index perturbation $n_{i}$, corresponding to a material gain $g_{0}=-2 n_{i} \omega / c$. The corresponding susceptibility perturbation is $\chi_{\text {pert }}=-n_{i}^{2}+i 2 n_{\mathrm{s}} n_{i}$. In practice, the value of the gain coefficient is controlled via the charge-carrier density. We 
notice that the ratio between the real and imaginary part of the susceptibility change is close to zero, corresponding to a nearzero value for the linewidth enhancement factor. In the results to be presented, we quantify the gain via the imaginary part of the refractive index; as examples, $n_{i}=-10^{-3}$ corresponds to a material gain of $g_{0}=81.1 \mathrm{~cm}^{-1}$ at $\lambda_{0}=1550 \mathrm{~nm}$ and $g_{0}=96.7 \mathrm{~cm}^{-1}$ at $\lambda_{0}=1300 \mathrm{~nm}$.

In the following we separately consider two waveguide lengths in order to illustrate and discuss two different physical effects. Section III A considers the case of relatively long waveguides, but weak material gain, where a strong backscattered signal may build up. This situation is shown to be accurately modeled by the derived coupled Bloch mode equations. Section III B considers short waveguides, but with strong material gain, in which case the perturbative treatment breaks down and the imaginary contribution to the refractive index changes the band structure.

\section{A. PhC waveguides with a long active section}

The length of the active $\mathrm{PhC}$ waveguide considered in this section is $L=100 a$. Numerically, such long waveguides are conveniently treated using the FMM that exploits the periodicity for obtaining the Bloch modes in both passive and active $\mathrm{PhC}$ sections. Specifically, it suffices to analyze a single unit cell (of length $a$ ), the eigensolutions of which are the Bloch modes [25,27], and the propagation through the full length $(L=100 a)$ is afterwards handled analytically via Bloch's theorem. Active-passive reflection and transmission are handled using a scattering matrix approach [39].

Figure 3 shows the transmitted and reflected power flux of an input cw light exciting a slow-light mode $[\omega a /(2 \pi c)=$

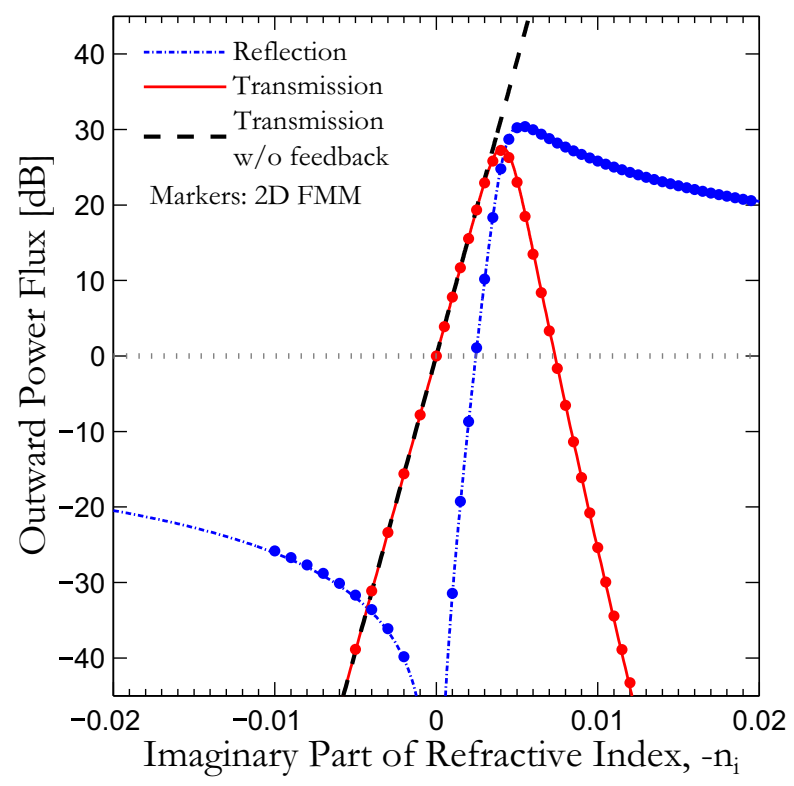

FIG. 3. (Color online) Power reflection (dash-dotted blue line) and transmission (solid red line) as functions of the imaginary part of the refractive index for the $\mathrm{PhC}$ structure in Fig. 1, with an active section of length $L / a=100$. A slow-light mode at $\omega a /(2 \pi c)=$ 0.2064 with a group index $n_{g z} \simeq 25$ [see Figs. 4(a) and 4(b)] is considered. Curves are results from the 1D coupled-wave analysis, with $\left|r_{1}\right|^{2}=\left|r_{2}\right|^{2}=0$, and markers are 2D FMM simulation results.
0.2064, $n_{g z} \simeq 25$, see Figs. 4(a) and 4(b)] as functions of the imaginary part of the refractive index.

The markers show the results based on 2D FMM simulations, while the solid lines are the results based on the solution of the 1D coupled Bloch wave equations. As is apparent, the two approaches agree well. In both cases, a nonzero value of the imaginary part of the refractive index leads to the buildup of a reflected signal. The dashed line shows the ideal slow-light enhanced device gain or absorption [7], $G=\exp \left(2 n_{g z} n_{\mathrm{s}} g_{0} \int_{0}^{L} \delta(z) d z\right)=\exp \left(\Gamma \frac{n_{g z}}{n_{\mathrm{s}}} g_{0} L\right)$, where $\Gamma$ is the confinement factor, the slope of which is proportional to the group index. It is seen that the simple slow-light enhanced gain expression in this case well accounts for the numerical results for absolute gain values up to about $25 \mathrm{~dB}$. Beyond that the transmission starts to decrease, due to the buildup of a strong back-reflected signal.

In the absorption regime $\left(-n_{i}<0\right)$, the increase of the material absorption leads to an increase of backscattered power flux, but the weak backscattered signal damped by material loss has a negligible effect on the transmitted power. On the other hand, in the regime of positive gain $\left(-n_{i}>0\right)$, the forward as well as backscattered signals are amplified and the effective length over which the waves interact is increased. As the material gain increases, the transmitted power reaches a maximum value and then steeply decreases.

We now briefly discuss the interpretation of the results in the context of the two different numerical techniques used, which effectively correspond to the use of different basis sets for representing the full solution. In the coupled Bloch mode approach, the total field is expanded on the Bloch modes of the passive waveguide [see Eqs. (2) and (6)]. The inclusion of material gain or absorption corresponds to a periodic perturbation of the imaginary part of the refractive index and induces coupling between the passive Bloch modes (DFB) that are no longer solutions to the full, perturbed problem. In the FMM approach, the local solutions are instead expanded on the active Bloch modes, i.e., the solutions when including the imaginary index perturbation. For an infinitely long active waveguide, these solutions are unidirectional Bloch modes with exponentially growing powers, which renders this concept unphysical. In the case of a finite-length active waveguide, the appearance of a back-reflected signal occurs due to the mismatch of the Bloch modes of the passive and active parts of the waveguide. This mode mismatch leads to a finite interface reflection coefficient and the appearance of Fabry-Pérot (FP) resonances that depend on the active region length. The equivalence of the DFB and FP pictures was previously discussed in the context of simple 1D optical grating structures [28].

Figures 4(c) and 4(d) show the transmission and reflection as a function of frequency for varying imaginary part of the refractive index. For a given frequency, as the imaginary part of the refractive index (material gain) increases, both transmission and reflection increase, reach a maximum, and then start to decrease. For certain combinations of frequency and material gain both the transmitted and reflected beams diverge. These points correspond to the fulfillment of the lasing oscillation condition for a laser with length given by the active region. The corresponding frequencies correspond to discrete values of the wave number, with the real part of the wave 

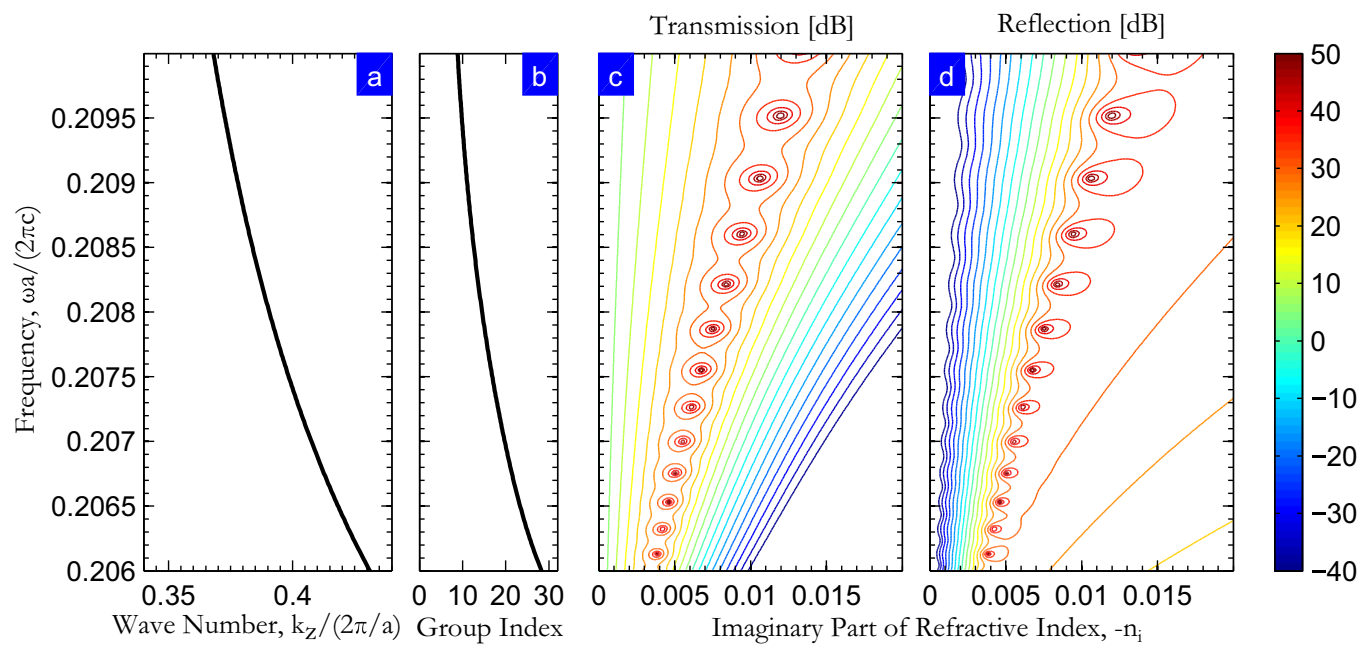

FIG. 4. (Color online) Propagation characteristics of a line-defect photonic-crystal waveguide with a gain section of finite length, $L=100 a$. (a) Dispersion curve and (b) group index, both of passive $\mathrm{PhC}$ waveguide Bloch mode. (c) Power transmission and (d) power reflection, both as functions of the imaginary part of the refractive index and frequency. The results in (c) and (d) are obtained by numerically solving the 1D coupled Bloch wave equations.

number fulfilling the condition

$$
\left(\frac{\pi}{a}-\operatorname{Re}\left(k_{z}\right)\right) 2 L=2 \pi m, \quad m=\text { integer. }
$$

Taking into account saturation of the gain medium due to stimulated emission, the divergences correspond to the onset of self-sustained lasing, with the imaginary part of the refractive index being clamped to its threshold value. Again, we notice the similarity of these results with the classical analyses of distributed feedback lasers [36] and active Bragg gratings [28].

For frequencies closer to the band gap, the resonances appear at smaller gain values, consistent with the enhancement of the gain per unit length due to slow-light propagation [7]. Notice that the gain per unit time inside the material is not changed, with the consequence, for lasers, that it is only the contribution of mirror losses to the laser threshold that is decreased when exploiting a slow-light mode.

\section{B. PhC waveguides with a short active section}

In this section we consider a short active $\mathrm{PhC}$ waveguide section, of length $L=20 a$, but allow for higher values of the material gain coefficient in order to explore the regime investigated in [23]. As the size of the 2D simulation domain decreases, it becomes feasible to solve the periodic optical waveguiding problem [40] in the frequency domain using the finite-element method (FEM) with numerically exact Bloch mode excitation and/or absorptive boundary conditions [41].

Figure 5 shows the transmitted and reflected power flux of an input cw light exciting a slow-light mode $[\omega a /(2 \pi c)=$ $0.2075, n_{g z} \simeq 18.7$ ] as a function of the imaginary part of the refractive index. The solid and dash-dotted lines are obtained from the 1D coupled-wave equations, while the markers are corresponding numerical results based on the 2D FEM. The results are shown in both logarithmic and linear scales in order to appreciate the differences between the two approaches. We see that the coupled-wave analysis reproduces most features seen in the full numerical FEM simulations for values of material gain and absorption coefficient up to $4000 \mathrm{~cm}^{-1}\left(\left|n_{i}\right|\right.$ up to 0.05$)$ at a wavelength of $1550 \mathrm{~nm}$, which is large compared to gain values typically obtained in semiconductors. For smaller gain values, the shorter device shows characteristics similar to the longer device, with the absolute gain being smaller for the same gain coefficient. We attribute the difference between the coupled-wave model and the full numerical result to a change of the effective gain coefficient appearing as the prefactor of $\Psi_{+}$and $\Psi_{-}$in the RHS of Eqs. (12) and (13). Thus, for a large absolute value of the imaginary part of the refractive index (large gain or absorption), the band structure is modified such that the group index is reduced and thereby also the slow-light enhanced gain and absorption. This is in agreement with the result obtained in [23], where the band structure of an infinitely periodic structure was analyzed. In that case, there is no effect of backscattering, since a unidirectional Bloch wave of the active structure is considered, but consistent with the results presented here the slow-light enhancement was observed to decrease with the absolute value of the material gain coefficient.

We interpret the gain-induced change of effective group index (slow-light enhancement factor) as follows. The origin of the photonic-crystal band gap is the destructive interference of multiply scattered waves. However, in the presence of gain (or absorption), the destructive interference is incomplete. For a defect photonic-crystal waveguide, this means that the band edge is smeared and the group index no longer diverges when approaching the band edge. In consequence, there is a gain-induced reduction of the group index, and thereby a gain-induced reduction of the slow-light enhancement of light-matter interaction.

In the example above we considered a relatively modest group index of $\simeq 18.7$, but when approaching the band edge further, the (passive) group index rapidly increases, and the saturation effect discussed above becomes more apparent, i.e., it sets in at lower values of the absolute gain coefficient. 


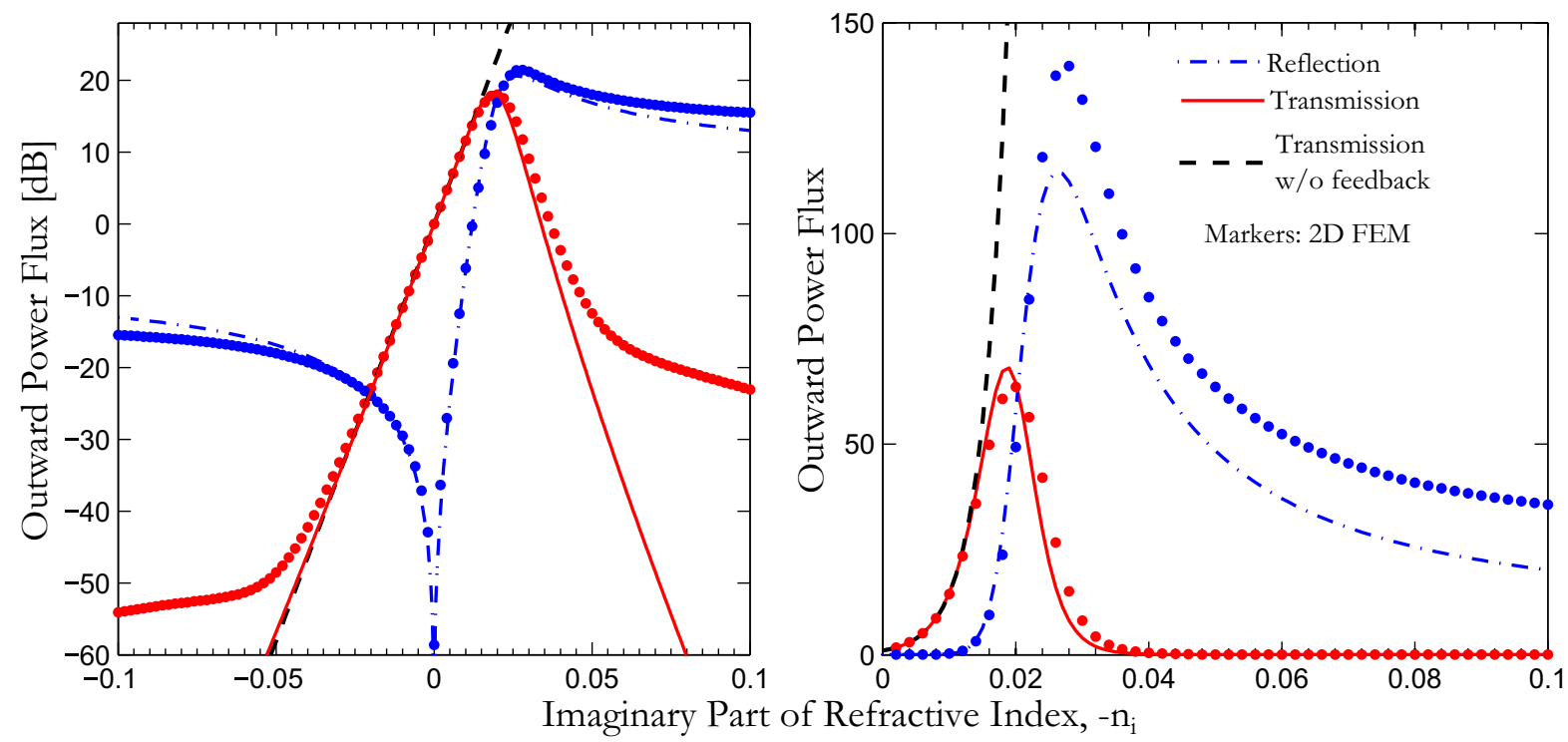

FIG. 5. (Color online) Power transmission and reflection in (a) decibel scale and (b) linear scale as functions of the imaginary part of the refractive index for the PhC structure in Fig. 1, with an active section of length $L / a=20$. The frequency is $\omega a /(2 \pi c)=0.2075$ with a corresponding group index of $n_{g z} \simeq 18.7$. Dash-dotted blue (reflection) and solid red (transmission) curves are 1D coupled-wave analysis results with zero passive-active interface reflections $\left(r_{1}=r_{2}=0\right)$, while markers are 2D FEM simulation results.

\section{CONCLUSIONS}

We have derived a set of coupled Bloch wave equations that are useful for simulating and understanding the properties of active photonic-crystal waveguides. The coupled Bloch wave model explains how slow-light effects can be used to enhance the absolute gain of a photonic-crystal waveguide of given length, an effect recently observed experimentally [7]. The model also shows, however, that the presence of material gain (an imaginary refractive index perturbation) induces distributed feedback effects, in addition to the slow-light enhancement of modal gain, which leads to the buildup of a backscattered field that limits the attainable gain. The coupled Bloch wave model is compared to numerical simulations based on the Fourier modal method as well as a frequency domain finite-element solver. In general, very good agreement between the different numerical approaches is found, although, depending on the approach used, the presence of a backscattered field calls for different, but consistent, interpretations. For very large values of the material gain (or absorption) coefficient, the band structure of the active photonic crystal itself is modified, leading to further changes of the slow-light enhancement, which is an effect not captured by the 1D coupled Bloch wave analysis.

Finally, we notice that the transparent and computationally efficient formulation of the coupled Bloch wave equations presented here allows for the inclusion of additional relevant effects. In [18], random disorder effects in passive structures were analyzed using a similar coupled Bloch wave analysis. With the recent observation of lasing in Anderson-localized modes due to disorder in active photonic-crystal waveguides [42], it appears natural to combine the two approaches.

\section{ACKNOWLEDGMENTS}

Y.C. expresses his gratitude to Professor Christian Hafner for helpful discussions on computational electromagnetics. Funding from Villum Fonden via the VKR Centre NATEC is gratefully acknowledged.
[1] S. McNab, N. Moll, and Y. Vlasov, Ultra-low loss photonic integrated circuit with membrane-type photonic crystal waveguides, Opt. Express 11, 2927 (2003).

[2] T. F. Krauss, Planar photonic crystal waveguide devices for integrated optics, Phys. Status Solidi A 197, 688 (2003).

[3] M. Notomi, A. Shinya, K. Nozaki, T. Tanabe, S. Matsuo, E. Kuramochi, T. Sato, H. Taniyama, and H. Sumikura, Low-power nanophotonic devices based on photonic crystals towards dense photonic network on chip, IET Circuits Devices Syst. 5, 84 (2011).

[4] F. Kish, D. Welch, R. Nagarajan, J. Pleumeekers, V. Lal, M. Ziari, A. Nilsson, M. Kato, S. Murthy, P. Evans, S. Corzine,
M. Mitchell, P. Samra, M. Missey, S. DeMars, R. Schneider, M. Reffle, T. Butrie, J. Rahn, M. Van Leeuwen, J. Stewart, D. Lambert, R. Muthiah, H.-S. Tsai, J. Bostak, A. Dentai, K.-T. Wu, H. Sun, D. Pavinski, J. Zhang, J. Tang, J. McNicol, M. Kuntz, V. Dominic, B. Taylor, R. A. Salvatore, M. Fisher, A. Spannagel, E. Strzelecka, P. Studenkov, M. Raburn, W. Williams, D. Christini, K. Thomson, S. Agashe, R. Malendevich, G. Goldfarb, S. Melle, C. Joyner, M. Kaufman, and S. Grubb, Current status of largescale InP photonic integrated circuits, IEEE J. Sel. Top. Quantum Electron. 17, 1470 (2011).

[5] J. P. Dowling, M. Scalora, M. J. Bloemer, and C. M. Bowden, The photonic band edge laser: A new approach to gain enhancement, J. Appl. Phys. 75, 1896 (1994). 
[6] T. Baba, Slow light in photonic crystals, Nat. Photonics 2, 465 (2008).

[7] S. Ek, P. Lunnemann, Y. Chen, E. Semenova, K. Yvind, and J. Mork, Slow-light-enhanced gain in active photonic crystal waveguides, Nat. Commun. 5, 5039 (2014).

[8] Y. Chen and J. Mørk, Theory of carrier depletion and light amplification in active slow light photonic crystal waveguides, Opt. Express 21, 29392 (2013).

[9] S. Matsuo, A. Shinya, T. Kakitsuka, K. Nozaki, T. Segawa, T. Sato, Y. Kawaguchi, and M. Notomi, High-speed ultracompact buried heterostructure photonic-crystal laser with $13 \mathrm{fJ}$ of energy consumed per bit transmitted, Nat. Photonics 4, 648 (2010).

[10] K. Nozaki, A. Shinya, S. Matsuo, Y. Suzaki, T. Segawa, T. Sato, Y. Kawaguchi, R. Takahashi, and M. Notomi, Ultralow-power all-optical RAM based on nanocavities, Nat. Photonics 6, 248 (2012).

[11] E. Kuramochi, K. Nozaki, A. Shinya, K. Takeda, T. Sato, S. Matsuo, H. Taniyama, H. Sumikura, and M. Notomi, Large-scale integration of wavelength-addressable all-optical memories on a photonic crystal chip, Nat. Photonics 8, 474 (2014).

[12] M. Calic, P. Gallo, M. Felici, K. A. Atlasov, B. Dwir, A. Rudra, G. Biasiol, L. Sorba, G. Tarel, V. Savona, and E. Kapon, PhononMediated Coupling of InGaAs/GaAs Quantum-Dot Excitons to Photonic Crystal Cavities, Phys. Rev. Lett. 106, 227402 (2011).

[13] E. Istrate and E. H. Sargent, Photonic crystal heterostructures and interfaces, Rev. Mod. Phys. 78, 455 (2006).

[14] J. E. Sipe, Vector $k \cdot p$ approach for photonic band structures, Phys. Rev. E 62, 5672 (2000).

[15] S. G. Johnson, P. Bienstman, M. A. Skorobogatiy, M. Ibanescu, E. Lidorikis, and J. D. Joannopoulos, Adiabatic theorem and continuous coupled-mode theory for efficient taper transitions in photonic crystals, Phys. Rev. E 66, 066608 (2002).

[16] J. E. Sipe, N. A. R. Bhat, P. Chak, and S. Pereira, Effective field theory for the nonlinear optical properties of photonic crystals, Phys. Rev. E 69, 016604 (2004).

[17] N.-C. Panoiu, J. McMillan, and C. W. Wong, Theoretical analysis of pulse dynamics in silicon photonic crystal wire waveguides, IEEE J. Sel. Top. Quantum Electron. 16, 257 (2010).

[18] M. Patterson, S. Hughes, S. Combrié, N. V. Q. Tran, A. De Rossi, R. Gabet, and Y. Jaouën, Disorder-Induced Coherent Scattering in Slow-Light Photonic Crystal Waveguides, Phys. Rev. Lett. 102, 253903 (2009).

[19] S. Mazoyer, J. P. Hugonin, and P. Lalanne, Disorder-Induced Multiple Scattering in Photonic-Crystal Waveguides, Phys. Rev. Lett. 103, 063903 (2009).

[20] Y. Chen and J. Mørk, Distributed feedback effects in active semiconductor photonic crystal waveguides, in Advanced Photonics Congress (Optical Society of America, Washington, DC, 2012), p. ITu2C.4.

[21] E. Mizuta, H. Watanabe, and T. Baba, All semiconductor low- $\delta$ photonic crystal waveguide for semiconductor optical amplifier, Jpn. J. Appl. Phys. 45, 6116 (2006).

[22] J. Mørk and T. R. Nielsen, On the use of slow light for enhancing waveguide properties, Opt. Lett. 35, 2834 (2010).

[23] J. Grgić, J. R. Ott, F. Wang, O. Sigmund, A.-P. Jauho, J. Mørk, and N. A. Mortensen, Fundamental Limitations to Gain
Enhancement in Periodic Media and Waveguides, Phys. Rev. Lett. 108, 183903 (2012).

[24] E. Noponen and J. Turunen, Eigenmode method for electromagnetic synthesis of diffractive elements with three-dimensional profiles, J. Opt. Soc. Am. A 11, 2494 (1994).

[25] G. Lecamp, J. P. Hugonin, and P. Lalanne, Theoretical and computational concepts for periodic optical waveguides, Opt. Express 15, 11042 (2007).

[26] L. Li, Use of Fourier series in the analysis of discontinuous periodic structures, J. Opt. Soc. Am. A 13, 1870 (1996).

[27] Sorting of the Bloch modes into modes in the $+z$ and $-z$ directions is nontrivial in the active $\mathrm{PhC}$ waveguide that supports both decaying and growing solutions; for more details see $\mathrm{J}$. R. de Lasson, P. T. Kristensen, J. Mørk, and N. Gregersen, Roundtrip matrix method for calculating the leaky resonant modes of open nanophotonic structures, J. Opt. Soc. Am. A 31, 2142 (2014).

[28] A. Yariv and A. Gover, Equivalence of the coupled-mode and Floquet-Bloch formalisms in periodic optical waveguides, Appl. Phys. Lett. 26, 537 (1975).

[29] L. Frandsen, A. Lavrinenko, J. Fage-Pedersen, and P. Borel, Photonic crystal waveguides with semi-slow light and tailored dispersion properties, Opt. Express 14, 9444 (2006).

[30] F. Wang, J. S. Jensen, J. Mørk, and O. Sigmund, Systematic design of loss-engineered slow-light waveguides, J. Opt. Soc. Am. A 29, 2657 (2012).

[31] T. P. White and A. Sukhorukov, Transition from slow and frozen to superluminal and backward light through loss or gain in dispersion-engineered waveguides, Phys. Rev. A 85, 043819 (2012).

[32] D. Michaelis, U. Peschel, C. Wächter, and A. Bräuer, Reciprocity theorem and perturbation theory for photonic crystal waveguides, Phys. Rev. E 68, 065601 (2003).

[33] H. Gersen, T. J. Karle, R. J. P. Engelen, W. Bogaerts, J. P. Korterik, N. F. van Hulst, T. F. Krauss, and L. Kuipers, Direct Observation of Bloch Harmonics and Negative Phase Velocity in Photonic Crystal Waveguides, Phys. Rev. Lett. 94, 123901 (2005)

[34] Y. Tanaka, Y. Sugimoto, N. Ikeda, H. Nakamura, K. Asakawa, $\mathrm{K}$. Inoue, and S. Johnson, Group velocity dependence of propagation losses in single-line-defect photonic crystal waveguides on GaAs membranes, Electron. Lett. 40, 174 (2004).

[35] S. Johnson and J. Joannopoulos, Block-iterative frequencydomain methods for Maxwell's equations in a planewave basis, Opt. Express 8, 173 (2001).

[36] H. Kogelnik and C. V. Shank, Coupled-wave theory of distributed feedback lasers, J. Appl. Phys. 43, 2327 (1972).

[37] S. Olivier, H. Benisty, C. Weisbuch, C. Smith, T. Krauss, and R. Houdré, Coupled-mode theory and propagation losses in photonic crystal waveguides, Opt. Express 11, 1490 (2003).

[38] B. Tromborg, H. Lassen, and H. Olesen, Traveling wave analysis of semiconductor lasers: Modulation responses, mode stability and quantum mechanical treatment of noise spectra, IEEE J. Quantum Electron. 30, 939 (1994).

[39] A. V. Lavrinenko, J. Lægsgaard, N. Gregersen, F. Schmidt, and T. Søndergaard, Numerical Methods in Photonics, Optical 
Sciences and Applications of Light (CRC Press, Boca Raton, FL, 2014).

[40] G. Bao, Finite element approximation of time harmonic waves in periodic structures, SIAM J. Numer. Anal. 32, 1155 (1995).
[41] C. Fietz, Absorbing boundary condition for Bloch-Floquet eigenmodes, J. Opt. Soc. Am. B 30, 2615 (2013).

[42] J. Liu, P. D. Garcia, S. Ek, N. Gregersen, T. Suhr, M. Schubert, J. Mørk, S. Stobbe, and P. Lodahl, Random nanolasing in the Anderson localized regime, Nat. Nanotechnol. 9, 285 (2014). 